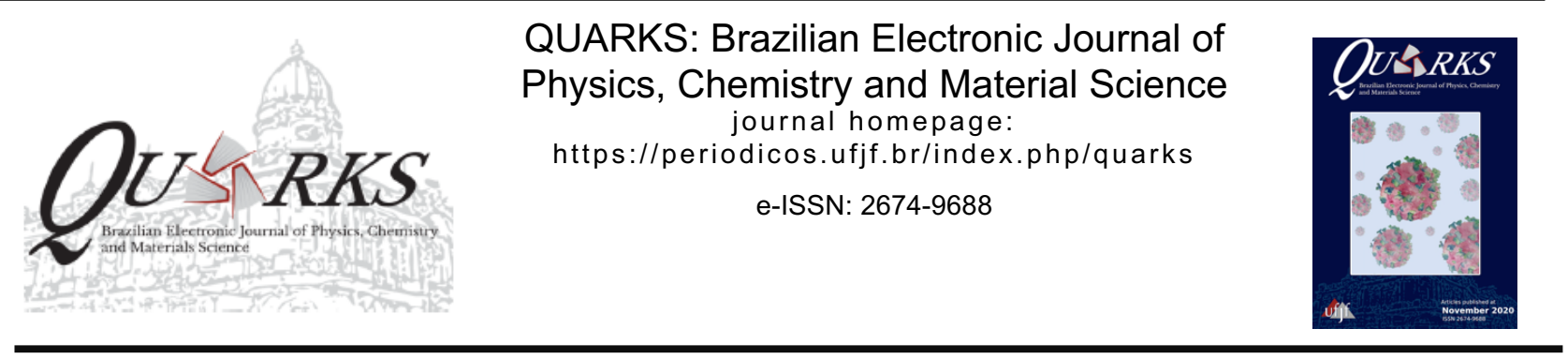

\title{
Quantitative behavior study of velocity, radius and topological charge on skyrmion/edge interaction dynamics on Co/Pt nanotrack
}

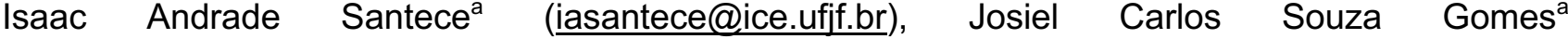 \\ (jcsgomes@fisica.ufjf.br), Danilo Toscano (danilo.toscano84@gmail.com), Maxwel Gama Monteiro \\ Jr. ${ }^{a}$ (maxweljr@gmail.com), João Paulo Almeida de Mendonça ${ }^{a}$ (jpamendonca@fisica.ufjf.br), \\ Clodoaldo Irineu Levartoski de Araujob (dearaujo@ufv.br), Fernando Sato ${ }^{a}$ (sjfsato@fisica.ufjf.br), \\ Sidiney de Andrade Leonela (sidiney@fisica.ufjf.br), Pablo Zimmermann Couraa (pablo@fisica.ufjf.br) \\ a Grupo de Física da Matéria Condensada, Laboratório de Simulação Computacional do Departamento, Departamento de \\ Física, Universidade Federal de Juiz de Fora, Rua José Lourenço Kelmer, s/n - São Pedro, 36036-900, Juiz de Fora, MG, \\ Brazil.

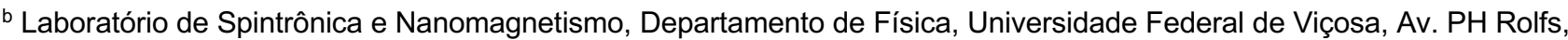 \\ s/n - Centro, 36580-900, Viçosa/MG.
}

Article history: Received: August 2020; Revised: September 2020; Accepted: October 2020. Available online: November 2020 https://doi.org/10.34019/2674-9688.2020.v3.31402

\begin{abstract}
Skyrmions are considered promising candidates to be the information carriers in the next generation of data storage and logic devices, due to its stability and easy control under the application of an electric current. For future technological applications in spintronic devices, it is important to study the properties behavior of these topological excitations during its movement on magnetic nanotracks, specially because in ferromagnetic materials they suffer a kind of magnus effect which tends to spell the skyrmion through the borders, preventing its transport throughout the nanotrack. We used micromagnetic simulations to study the dynamics of a skyrmion on a magnetic nanotrack induced by a spin polarized electric current. We considered thin magnetic nanotrack made of cobalt and platinum multilayers, whose magnetic state is perpendicular to the track plane and contain a single Néel-type Skyrmion. To describe this magnetic system, we used a Hamiltonian containing exchange, Dzyaloshinskii-Moriya, perpendicular magnetic anisotropy and dipole-dipole interactions. In our study we observed the well-known Skyrmion Hall effect and changes in the structure of the skyrmion when it approaches of the border. This alteration can be measured by determining the radius and the topological charge of the Skyrmion. Our simulation results show that both the radius and the topological charge decrease when it approaches of the border. Our study also demonstrates that the skyrmion-border interaction is repulsive, but there is a minimum distance from the border at which the interaction becomes attractive. If the skyrmion exceeds this critical position $\mathrm{y}_{\mathrm{c}}$, it will be attracted and annihilated at the border of the nanotrack. We also performed simulations to obtain the limit value $\mathrm{j}_{\mathrm{c}}$ of the applied current density that the skyrmion can be transported along of the nanotrack without escaping from the side edge. From a technological point of view for possible applications in spintronic devices, the estimate of $j_{c}$ is of crucial importance.
\end{abstract}

Keywords: Ferromagnetic Spintronics, Magnetization Dynamics, Micromagnetic Simulations, Skyrmions.

\section{Introduction}

Since the random access memories implementation by IBM in the 1950s, several different changes have been done to increase the access speed of storage hardware. Nowadays there is a search for new storage hardwares using nanostructured magnetic samples to decrease the access time and increase the storage capacity on those devices [1-3]. Such samples can be manufactured in different shapes using materials such as cobalt and Permalloy alloy. These samples can present many types of domain regions (exotic magnetic structures or quasiparticles) such as vortices, skyrmions and domain walls, depending on the material, shape, and sample size. These topological excitations enable us engineer spintronic devices [4,5] where each magnetic 
structure can be considered as a basic information unit (bit) $[6,7]$. In this work we study magnetic skyrmions, which are likely to be the next generation of storage devices and data logic [8].

Skyrmions were presented by Tony $H$. R. Skyrme in a particle physics study, in which he developed a nonlinear field theory for interacting pions and showed that topologically stable configurations occur as particles solutions $[9,10]$. The concept of these topologically protected configurations were extended to condensed matter physics and nowadays skyrmions can be found in nanoscaled magnetic thin films [11-16]. Magnetic skyrmions are considered topologically protected and relatively stable because they cannot be continuously deformed until reach another magnetic state [17]. In magnetic materials, skyrmions were initially observed experimentally at low temperature and under external magnetic fields [18-20]. Recently, they have already been stabilized at room temperature without the application of a magnetic field [21-25].

Skyrmions appear in magnetically ordered systems that feature Dzyaloshinskii-Moriya (DM) coupling [26-29]. This coupling can appear intrinsically in chiral magnets (compounds of transition metals with the noncentrosymmetric B20type structure, for example) [18] or may be induced in magnetic multilayer systems with low inversion symmetry and strong spin-orbit coupling (in $\mathrm{Co} / \mathrm{Pt}$ multilayers, for example) [21]. Nanostrips made of Co/Pt multilayers allow the emergence of Néel-type skyrmions [21]. In chiral magnets, skyrmions are stabilized at low temperature under the application of an external magnetic field [18]. Skyrmions excited in Co/Pt nanotrack can be stabilized at room temperature and, due to the strong spin-orbit coupling at the interface and the strong magnetocrystalline anisotropy, do not require the application of an external magnetic field [21]. Individual skyrmions can be written or deleted on a ultrafine magnetic film (nanotrack) in a controlled manner [21] and can be easily moved by applying low polarized spin current densities $[15,30]$. This last characteristic, aside of the non-destructive properties described above, points skyrmions as the most prominent structure to replace domain walls in spintronic devices.

Under the influence of a spin polarized current along the ferromagnetic nanotrack, skyrmions suffer a transverse deflection in their motion, similar to the Hall effect that occurs with charged particles in the presence of magnetic field. This phenomenon, known as the skyrmion Hall effect, occurs due to the Magnus force that drive them towards one of the nanotrack edges, where it will be annihilated. Then, to a narrower track, the distance traveled by the skyrmion is dramatically reduced $[16,31,32]$. This effect is a problem from the technological point of view to the development of spintronic devices based on skyrmion transport.

Various strategies can be used to suppress the skyrmion Hall effect. One of these strategies is to modify the magnetic medium by including defects, such as magnetic impurities. Magnetic defects are variations on the magnetic properties of the medium and can be intentionally incorporated in nanomagnets in order to create traps for vortex [33], domain walls [34,35] and skyrmions [36]. Example can be cited, as applying $\mathrm{Ga}^{+}$ion beam to the multilayer interface can increase the coercivity of the implanted region [37]. The region with the highest coercivity results in pinning region for the domain wall [38]. Also, the Dzyaloshinskii-Moriya interaction can be changed by breaking cobalt platinum interface symmetry with different platinum layer thicknesses $[39,40]$.

In previous works, our group observed that the local decrease (increase) of the exchange stiffness constant acts as an attraction (repulsion) pinning center to a vortex, domain wall or skyrmion $[33,34,41,42]$. In two recent works [43,44] we showed other three ways to build magnetic skyrmion traps using magnetic defects, as variation in magnetocrystalline anisotropy, in DM interaction and in dipolar constant. Recently, we proposed several ways of suppression the skyrmion Hall effect in ferromagnetic nanotracks with their magnetic properties strategically modified [44].

Another alternative to suppress the Hall effect of skyrmion is to replace the nanotrack ferromagnetic material by an antiferromagnetic material, that is, using a real antiferromagnetic nanotrack [45-47]. In a recent work we investigated the controllability of the skyrmion position in antiferromagnetic nanotracks with their magnetic properties modified spatially [48].

In this work we performed micromagnetic simulations to study the dynamics of a skyrmion on a magnetic nanotrack made of Co/Pt multilayers, induced by a spin polarized current. The behavior of skyrmions in this type of nanostructure is still being 
studied because stable zero-field skyrmions are more suitable for technological application in ultralow energy devices. We used a bioinspired algorithm, known in the literature as the Frog Method [49], to accurately determine the position of the skyrmion during its dynamics. Changes in the skyrmion radius and topological charge were observed in our simulations. Finally, we also studied the interaction energy between the skyrmion and the nanotrack edge.

\section{Model and Methodology}

In this work we considered a thin magnetic multilayer of $\mathrm{Co} / \mathrm{Pt}$ containing a single Neél-type Skyrmion, whose equilibrium magnetization configuration is perpendicular to the track plane. To describe this magnetic system, we used a Hamiltonian containing exchange, DzyaloshinskiiMoriya, perpendicular magnetic anisotropy and dipole-dipole interactions:

$$
\begin{aligned}
& H=J\left\{-\sum_{<i, j>} \widehat{m}_{i} \cdot \widehat{m}_{j}-\frac{K}{J} \sum_{i}\left(\widehat{m}_{i} \cdot \hat{z}\right)^{2}+\frac{D}{J} \sum_{<i, j>} \widehat{m}_{i} \cdot\left(\widehat{m}_{j} \times \widehat{d_{l J}}\right)\right. \\
& \left.+\frac{M}{J} \sum_{i, j} \frac{\left[\left(\widehat{m}_{i} \cdot \widehat{m}_{j}\right)-3\left(\hat{m}_{i} \cdot \hat{r}_{i j}\right)\left(\hat{m}_{j} \cdot \hat{r}_{i j}\right)\right]}{\left(\left|\vec{r}_{i j}\right| / a\right)^{3}}\right\}
\end{aligned}
$$

where $\widehat{m}_{i}$ and $\widehat{m}_{j}$ are unit vectors representing the magnetic moments located at the $i$ and $j$ sites. In our simulations we use the micromagnetic approach $[33,34,42,50]$, which means partition the nanotrack into cubical working cells containing many atoms. The work cell size has a volume $V_{\text {cell }}=a^{3}$, where $a$ is the lattice parameter ( $a$ is greater than unit cell parameter $a_{0}$ ) and $\vec{m}_{k}=$ $\left(M_{s} V_{\text {cell }}\right) \hat{m}_{k}$, is the effective magnetic moment where $M_{S}$ is the saturation magnetization constant.

The first term of the equation (1) describes ferromagnetic coupling, once $J>0$. Due to the exchange interaction short range, the summation is over the nearest magnetic moment pairs $\langle i, j\rangle$. The micromagnetic exchange constant is given by $J=2 a A$, where $A$ is the exchange stiffness constant.

The second term of the equation (1) describes the uniaxial magnetocrystalline anisotropy, with preferential axis in the z-direction. This constant is $K=a^{3} k$, where $k$ is the magnetocrystalline anisotropy constant. The third term of the equation (1) describes the Dzyaloshinskii-Moriya interaction and, due to the short range of this interaction, the summation is over the nearest magnetic moment pairs $\langle i, j\rangle$. The versor $\hat{d}_{i j}$ depends on the type of magnetic system considered. Since we are considering multi-layer magnetic systems, the relationship between the versors is $\hat{d}_{i j}=\hat{r}_{i j} \times \hat{z}$, where $\hat{z}$ is a versor perpendicular to the multilayer surface and $\hat{r}_{i j}$ is a versor that joins the $i$ and $j$ sites in the same layer [43]. The micromagnetic constant $D=a^{2} d$, where $D$ is the Dzyaloshinskii-Moriya interaction strength.

The last term of the equation (1) represents the magnetic moments dipole-dipole coupling. Here, the ratio $\frac{M}{J}=\frac{1}{4 \pi}\left(\frac{a}{\lambda}\right)^{2}$, where $\lambda$ is the exchange length that depends on the magnetic material. The strength of the magnetic interactions, $J, d, K$ and $M$ have the same dimension (energy unity).

We choose the typical parameters for $\mathrm{Co} / \mathrm{Pt}$ [15]: exchange stiffness constant $A=1.5 \times 10^{-11} \mathrm{~J} / \mathrm{m}$, saturation magnetization $M_{s}=5.8 \times 10^{5} \mathrm{~A} / \mathrm{m}$, magnetocrystalline anisotropy $k=6.0 \times 10^{5} \mathrm{~J} / \mathrm{m}^{3}$ and Dzyaloshinskii-Moriya interaction strength $d=$ $4.0 \times 10^{-3} \mathrm{~J} / \mathrm{m}^{2}$.

The cells size a (cell lattice parameter) is chosen by analyzing three characteristic lengths relevant of the magnetic system: the exchange length $\lambda=$ $\sqrt{\frac{2 A}{\mu_{0} M_{S}^{2}}} \approx 8.42 \mathrm{~nm}$, the Bloch domain wall width $\Delta=$ $\sqrt{\frac{A}{k}} \approx 5.00 \mathrm{~nm}$ and the length associated with Dzyaloshinskii-Moriya interaction $\xi=\frac{2 A}{d} \approx$ $7.50 \mathrm{~nm}$. In our simulations, we used for lattice parameter $a=2 \mathrm{~nm}$ which is smaller than the smallest characteristic lengths $\Delta$ and $V_{\text {cel }}=$ $(2 \times 2 \times 2) n m^{3}$.

The magnetization dynamics behavior was 
obtained by integrating a version of the LandauLifshitz-Gilbert equation (LLG) which includes the spin torque effect. The dimensionless LLG equation, considering the spin polarized current applied along the $\mathrm{x}$-direction can be described by [51]:

$$
\begin{gathered}
\frac{\partial \widehat{m}_{i}}{\partial \tau}=-\frac{1}{\left(\alpha^{2}+1\right)}\left\{\widehat{m}_{i} \times \vec{h}_{i}^{e f f}+\alpha \widehat{m}_{i} \times\left(\widehat{m}_{i} \times \vec{h}_{i}^{\text {eff }}\right)\right. \\
\left.+\frac{1}{\left(\beta^{2}+1\right)}\left(\frac{v_{j}}{a \omega_{0}}\right)\left[(\beta-\alpha) \widehat{m}_{i} \times \frac{\partial \widehat{m}_{i}}{\partial \chi}+(\alpha \beta+1)\left(\frac{v_{j}}{a \omega_{0}}\right) \widehat{m}_{i} \times\left(\widehat{m}_{i} \times \frac{\partial \widehat{m}_{i}}{\partial \chi}\right)\right]\right\}
\end{gathered}
$$

where $\quad \vec{h}_{i}^{e f f}=-J^{-1} \frac{\partial H}{\partial \widehat{m}_{i}}$ is the dimensionless effective field located at the cell $i$, containing individual contributions from the exchange, anisotropy, Dzyaloshinskii-Moriya and dipolar fields. Notice that the first two terms describe the precession and damping torques, whereas the last two terms describe the torque due to the injection of the spin polarized electric current [7]. Typical nondimensional parameters for cobalt have been used in our simulations [51]: the Gilbert damping parameter $\alpha=0.1$ and the degree of nonadiabaticity $\beta=0.35$. The influence of the ratio $(\beta / \alpha)$ on the dynamics of magnetic domain walls has already been investigated [53-55]. Here, $\chi$ is dimensionless space coordinate, $\tau$ is dimensionless time coordinate and the relation with the real coordinates is given by $\Delta \chi=\Delta x / a$ and $\Delta \tau=\omega_{0} \Delta t$, where $\omega_{0}=\left(\frac{\lambda}{a}\right)^{2} \gamma \mu_{0} M_{s}$ is a scale factor with inverse time dimension, with $\gamma \approx 1.76 \times 10^{11}(T$. $s)$ is the electron gyromagnetic ratio. In or simulation $\omega_{0} \approx 2.27 \times 10^{12} \mathrm{~s}^{-1}$. The product $\left(a \omega_{0}\right)$ has dimension of velocity and the term $v_{j}=$ $j_{e} \eta P$, where $j_{e}\left(\vec{j}_{e}=j_{e} \hat{x}\right.$, so that $\left.\vec{v}_{j}=v_{j} \hat{x}\right)$ is the $\mathrm{x}$ component of the electric current density vector and $P=0.7$ is the rate of spin polarization [52], which amounts to those reported in cobalt nanowires of similar thicknesses. For cobalt, the constant $\eta \equiv \frac{g \mu B}{2 e M_{s}} \approx 9,99 \times 10^{-11} \mathrm{~m}^{3} / C$, where $g \approx 2$ is the Lande factor, $\mu_{B}$ is the Bohr magneton, and $e$ is the elementary positive charge. The time step $\Delta \tau=0.01$ used in the numerical simulations corresponds to $\Delta t=4.39 \times 10^{-15} \mathrm{~s}$.

Once Co/Pt multilayers allow the emergence of Néel-type skyrmions (hedgehog-type configuration), the magnetic configuration with a single skyrmion here been chosen as initial condition, by considering an analytical solution in which a Néel skyrmion is placed in a suitable position on the nanotrack. A description of the analytical model for skyrmions in nanotracks can be found in reference [44]. In the absence of a magnetic field or spin polarized current, the integration of the LLG equation leads to the stable configuration. Such relaxation in micromagnetic simulations allows the adjustment not only of the skyrmion radius but also its out-of-plane magnetization. The stable configuration obtained in this way has been used as initial configuration in other simulations, in which a spin polarized current was applied to move the skyrmion on nanotrack.

There are a number of works in the literature that have proposed ways to map the skyrmion position. In this work we investigate and compare three methods: the Magnetization Average Method $[55,56]$, the Charge Method [56,57] and the Frog Method [49]. In order to elect the most accurate method, we track the skyrmion position for each simulation step. When plotting the magnetic configuration together with graphs of the skyrmion trajectory we can validate the above-mentioned methods.

In the Magnetization Average Method, we can track its position $x_{(A v)}$ and $y_{(A v)}$ by a simple calculation:

$$
\begin{array}{r}
x_{(A v)}=\frac{\int x(i)\left[m_{z}(i)-1\right] d V}{\int\left[m_{z}(i)-1\right] d V}, \\
y_{(A v)}=\frac{\int y(i)\left[m_{z}(i)-1\right] d V}{\int\left[m_{z}(i)-1\right] d V}
\end{array}
$$


where $x(i)$ and $y(i)$ are the lattice sites coordinates and $m_{z}(i)=\widehat{m}_{i} \cdot \hat{z}$. In the Charge Method, skyrmion position $x_{(s)}$ and $y_{(s)}$ can be calculated by:

$$
\begin{aligned}
& x_{(s)}=\frac{\int x(i) s d V}{\int s d V}, \\
& y_{(s)}=\frac{\int y(i) s d V}{\int s d V}
\end{aligned}
$$

where $x(i)$ and $y(i)$ are the lattice site coordinates and $s$ is the topological density that arises from the topological charge $(S)$ calculation:

$$
\begin{gathered}
S=\frac{1}{4 \pi} \int \widehat{m} \cdot\left(\frac{\partial \widehat{m}}{\partial x} \times \frac{\partial \widehat{m}}{\partial y}\right) d x d y=\frac{1}{4 \pi} \int s d x d y \\
s=\widehat{m} \cdot\left(\frac{\partial \widehat{m}}{\partial x} \times \frac{\partial \widehat{m}}{\partial y}\right) \quad \text { (5) }
\end{gathered}
$$

where $\widehat{m}$ are the normalized magnetization vectors.

A bioinspired algorithm was used in the Frog Method. These types of algorithms are based on natural systems used to solve, for example, optimization problems. Optimization problems are those where the maximum and minimum values of functions are defined in a predefined interval. The Frogs method used in this work is based on minimizing a function that represents the total error in predicting the value of $m_{z}(i)$. The $m_{z}(i)$ represents a skyrmion configuration in a given region. This method works like a "skyrmion-hunt". The reference [49] describes the methodology used in the Frog Method.

In micromagnetic simulations, we have used our own computational code, which has been used in several works of our group. We have implemented in this program the fourth-order predictor-corrector method to solve numerically the equation (2).

We started by generating a fixed size magnetic Co/Pt nanotrack, with length $L=500 \mathrm{~nm}$, width $W=80 \mathrm{~nm}$ and thickness $T=2 \mathrm{~nm}$, containig an isolated Néel-type Skyrmion as magnetic configuration, whose profile is represented in figures 1 and 2 .

Integrating the LLG equation without the spin polarized electric current application, we obtained the magnetic equilibrium configurations. In the Table I are presented the material parameters used in micromagnetic simulations.

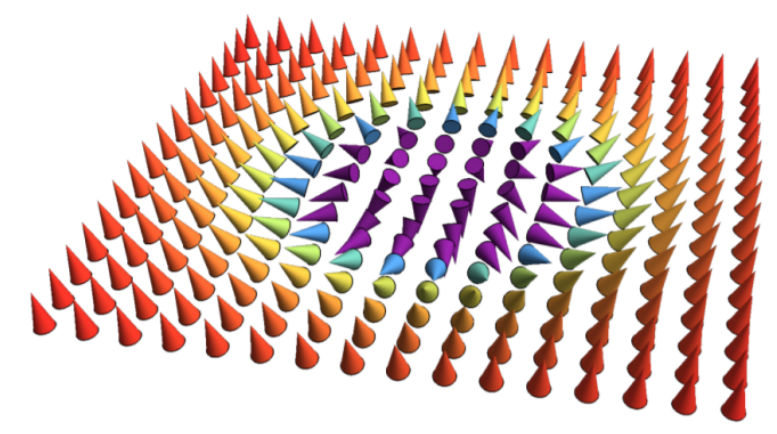

Figure 1- Profile of a Néel-type skyrmion located in the $x y$ plane. The small cones represent the magnetic moments $\widehat{\boldsymbol{m}}$ of each cell (site). The Néel type skyrmion has a hedgehog-like configuration and the central magnetic moment has an opposite direction to the magnetic moments of the edge.

\section{DHWWAPRAOWASA}

Figure 2- Profile of a diametrical section of the Néeltype skyrmion located in the $x \boldsymbol{y}$ plane. 
TABLE I - Parameters used in the micromagnetic simulations.

\begin{tabular}{l|c}
\hline Quantity & Value \\
\hline Exchange stiffness $(A)$ & $1.5 \times 10^{-11} \mathrm{~J} / \mathrm{m}$ \\
Saturation magnetization $\left(M_{S}\right)$ & $5.8 \times 10^{5} \mathrm{~A} / \mathrm{m}$ \\
Uniaxial anisotropy constant $(K)$ & $1.2 \times 10^{6} \mathrm{~J} / \mathrm{m}^{3}$ \\
Dzyaloshinskii-Moriya constant $(D)$ & $4.0 \times 10^{-3} \mathrm{~J} / \mathrm{m}^{2}$ \\
Work cell size $(a)$ & $2.0 \mathrm{~nm}$ \\
Damping parameter $(\alpha)$ & 0.1 \\
Degree of non-adiabaticity $(\beta)$ & 0.35 \\
Spin polarization of the current $(P)$ & 0.7 \\
\hline
\end{tabular}

First, we consider as the initial magnetic equilibrium configuration the skyrmion located in the center of the Co/Pt nanotrack, where is the origin of the coordinate system, as shown in figure 3 .

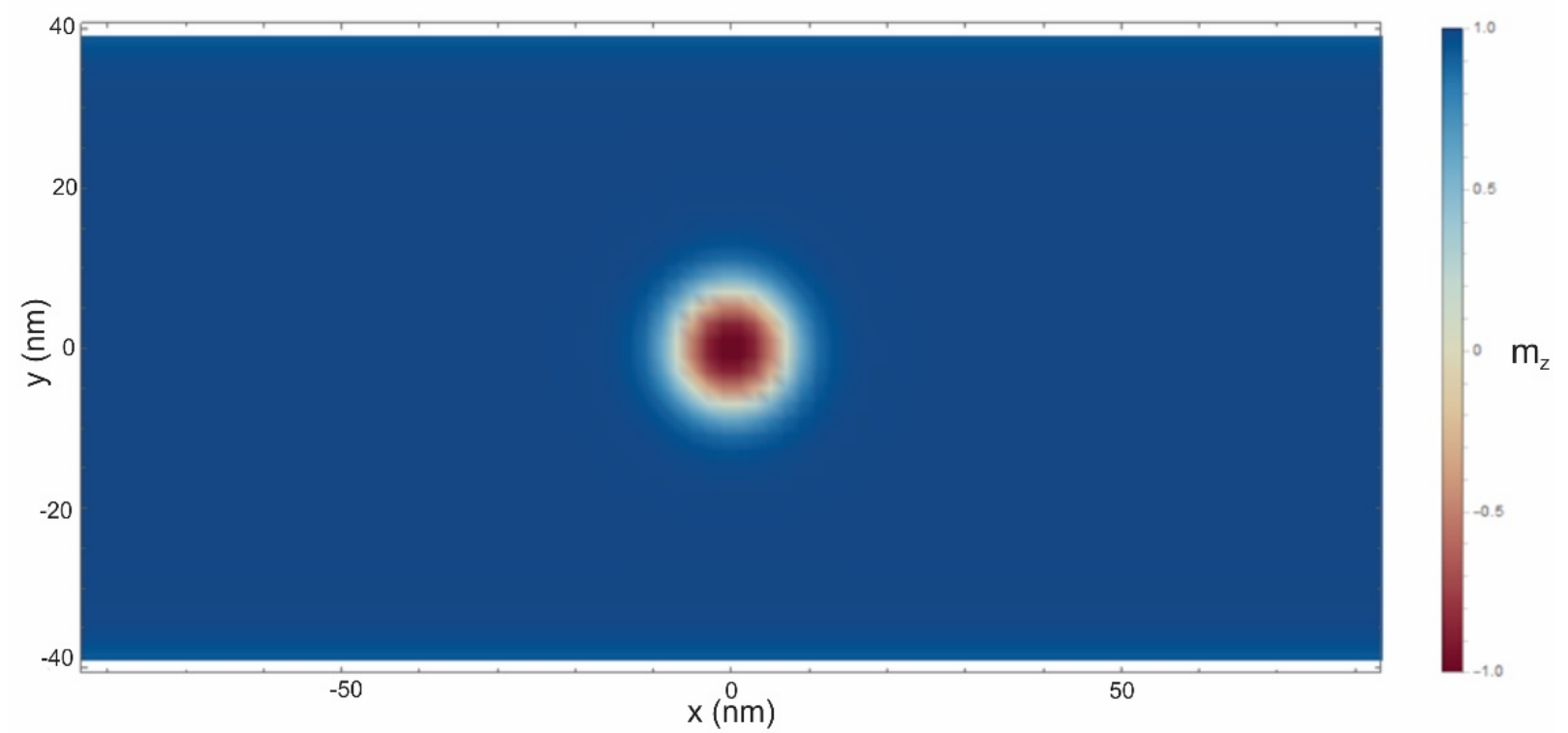

Figure 3- Schematic view of a rectangular nanotrack in the $\boldsymbol{x y}$ plane hosting a single magnetic skyrmion in the center, where is the origin of the coordinate system. The figure highlights that the majority of the nanowire's magnetic moments is going out of the plane of the figure (+z direction) except at the core of the skyrmion (red region), where they are pointing in the opposite direction. For simplicity, the cones representing magnetic moments are not displayed in the figure. 


\section{Results and Discussion}

The skyrmion position is changed by applying spin polarized electric current in the direction of the nanotrack major axis (x-axis), with current density $j_{e}=1 \times 10^{12} \mathrm{~A} / \mathrm{m}^{2}$. In figure 4 is shown the trajectory of the skyrmion in the $x y$ plane during current-induced displacements similar to the particles Hall effect even having not electrical charge. This effect is an obstacle to its application to memory devices and in restricted geometries the skyrmion reaches the nanotrack edges very fast and is destroyed. Thus, the distance that a skyrmion can be moved drastically reduces as the nanotrack becomes narrower [31,32].
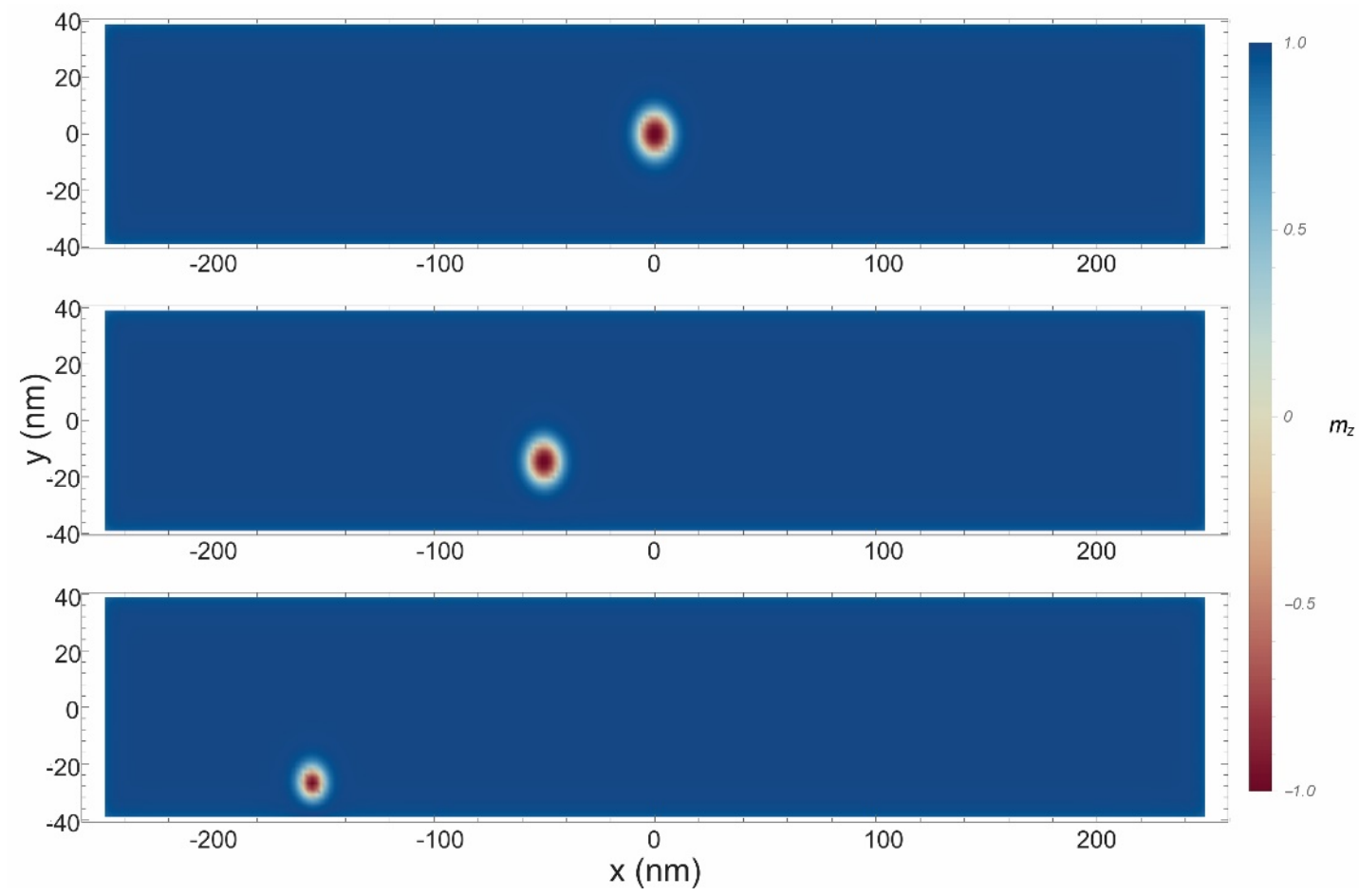

Figure 4- Successive snapshots of the current-induced skyrmion displacements in the nanotrack. It can be observed a transverse displacement similar to the particles Hall effect, called skyrmion Hall Effect.

Figure 5 shows the trajectories of the skyrmion during the transversal displacement shown in figure 4 obtained by the three methods: the Average Magnetization Method $(A v)$, the Charge Method(s) and the Frog Method(Frog). The $x_{s k}$ and $y_{s k}$ are the $x$ and $y$ coordinates of the center of the skyrmion, respectively. We can see that the result obtained by Average Magnetization Method shows a deviation in the trajectory when the skyrmion approaches the edge, failing to correctly describe the skyrmion's position. The Charge Method and the Frog Method have very similar trajectories.

Figure 6 shows successive snapshots of the skyrmion dynamics on the nanotrack and the three trajectories obtained by the Average Magnetization Method, Charge Method and the Frog Method. The skyrmion is represented by the orange circle. The trajectory generated by the Frog Method is the one that best fits the displacement of the center of the skyrmion. The Charge Method, due to the translation symmetry breaking at the edge, shows a small deviation in the trajectory. 


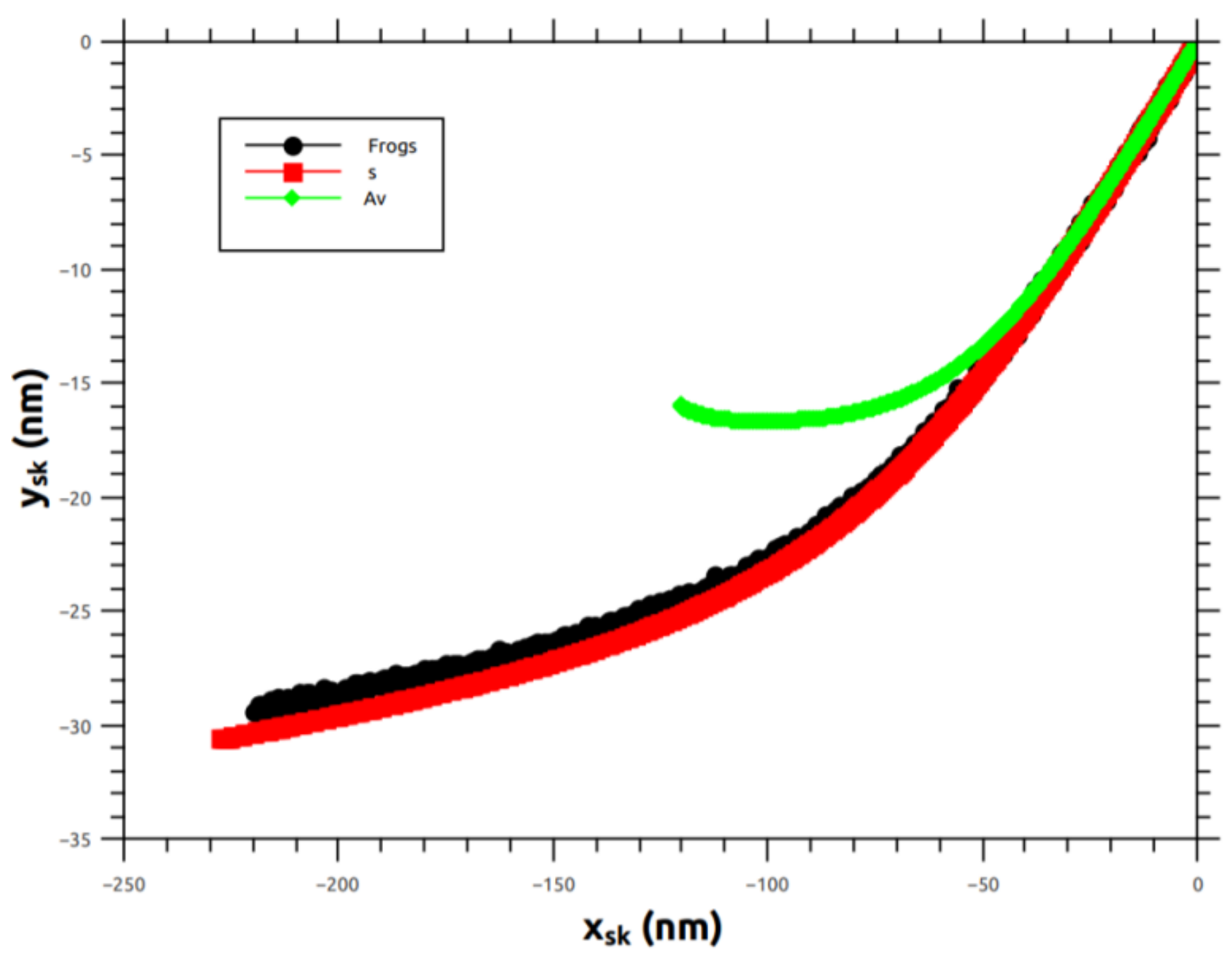

Figure 5- Trajectories in the xy-plane of the skyrmion during the transversal displacement shown in figure 4, obtained by the three methods: the Average Magnetization Method $(\boldsymbol{A} \boldsymbol{v})$, the Charge Method (s) and the Frog Method(Frog). The $x_{s k}$ and $\boldsymbol{y}_{\boldsymbol{s} \boldsymbol{k}}$ are the $\boldsymbol{x}$ and $\boldsymbol{y}$ coordinates of the center of the skyrmion, respectively. 


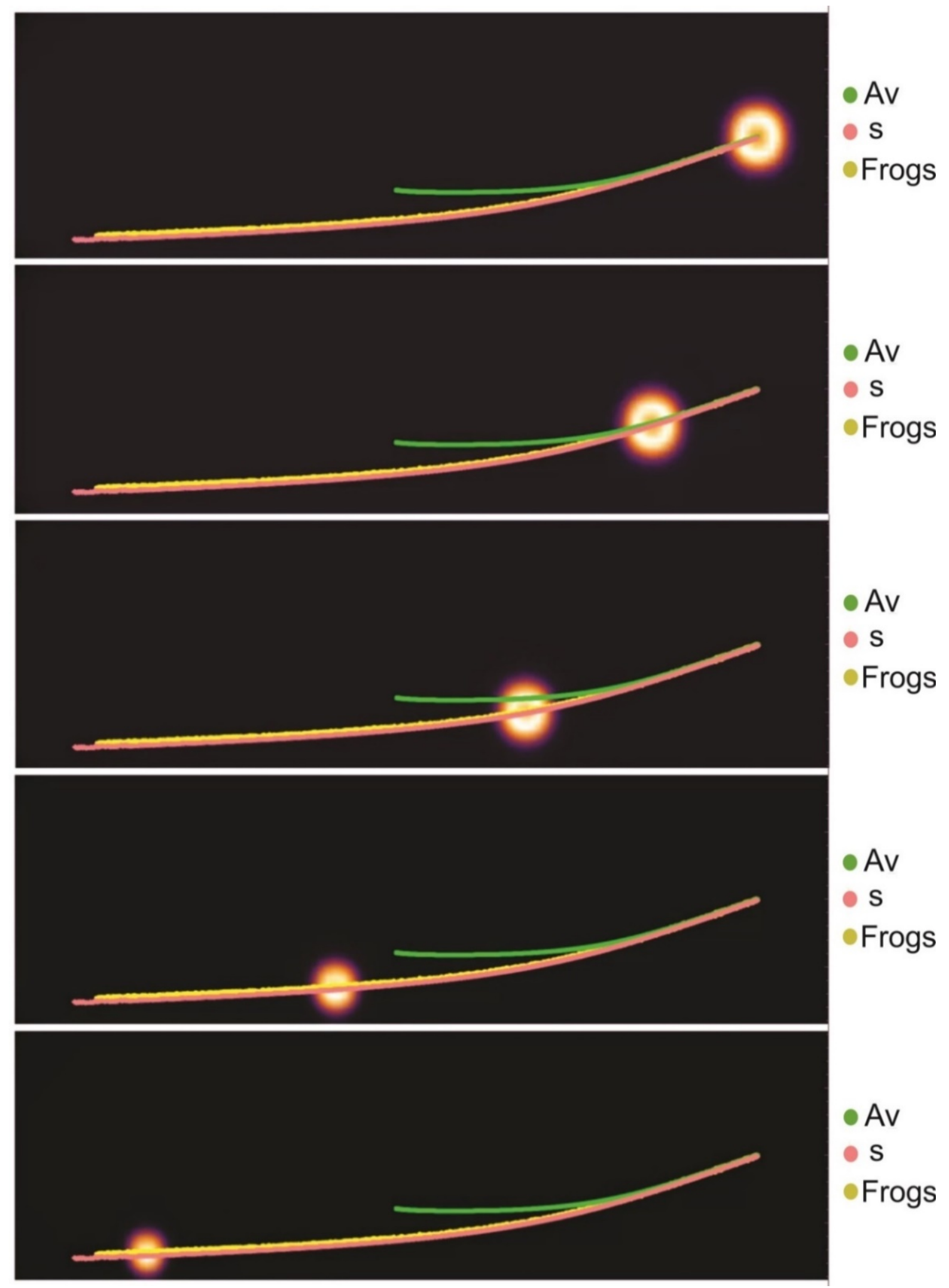

Figure 6- Successive snapshots of the skyrmion movement in the nanotrack accompanied by the representation of the three trajectories obtained by the Average Magnetization Method (pink line), the Charge Method (green line) and the Frog Method (yellow line), respectively. The skyrmion is represented by the orange circle. 
We also analyzed the behavior of the components $v_{x}(t)$ and $v_{y}(t)$ of the skyrmion velocity during the displacement (see figure 7). To obtain the behavior of $v_{x}(t)$ and $v_{y}(t)$ shown in figure 7, we derive in relation to time $t$ the $x_{s k}(t)$ and $y_{s k}(t)$ calculated by the Frog Method.

We observed an increase in longitudinal velocity $\left(v_{x}\right)$ and a decrease in transverse velocity $\left(v_{y}\right)$ acquired by the skyrmion when approaching the edge, which qualitatively agrees with the theoretical results of the reference [58]. Analyzing the first 300 steps of the dynamics and using linear regression, we can estimate the initial velocity components $v_{0 x}$ and $v_{0 y}$ of the skyrmion. We obtain the values $v_{0 x}=(64.45 \pm 0.02) \mathrm{m} / \mathrm{s}$ and $v_{0 y}=(19.16 \pm$ $0.02) \mathrm{m} / \mathrm{s}$ for these components.

When the skyrmion approaches the side edge its radius $r_{s}$ and topological charge $S$ are changed. The radius $r_{s}$ is defined as the distance between the magnetic moment of the center of the skyrmion $(-z$ direction) and the first magnetic moment with an angle of $90^{\circ}$ with respect to it (see the figure 8).

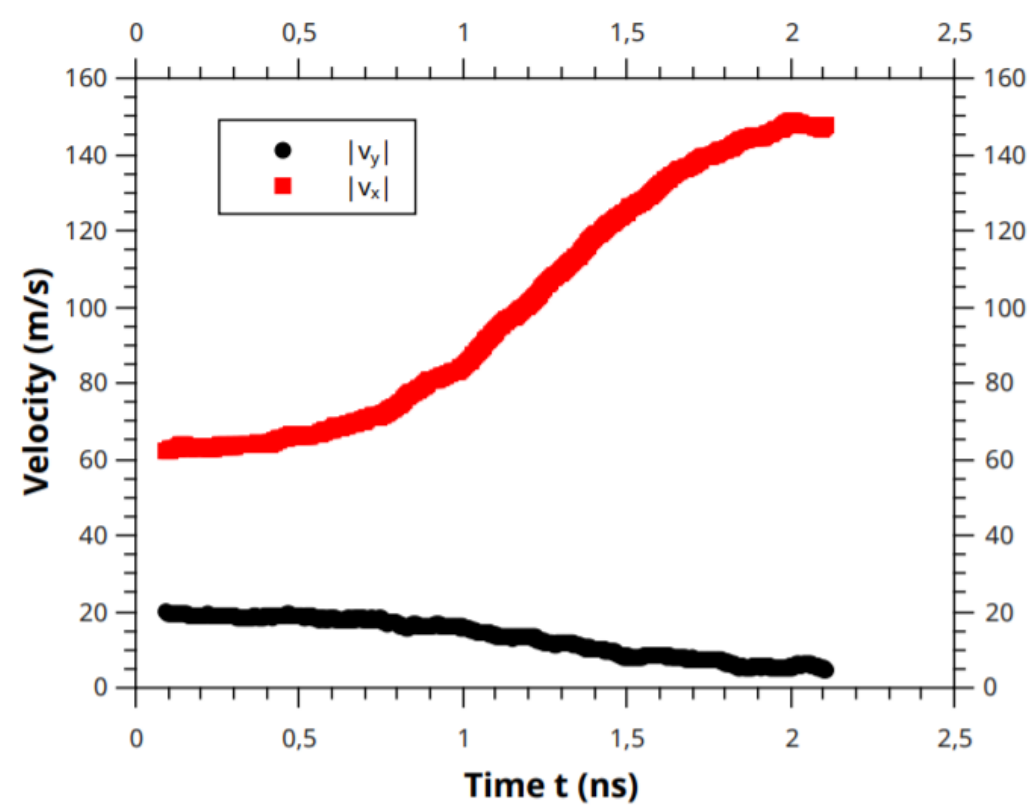

Figure 7- Module of the $v_{x}$ and $v_{y}$ components of the skyrmion velocity as a function of time $t$, during the displacement shown in Figure 4. The $v_{x}(t)$ and $v_{y}(t)$ were calculated using $x_{s k}(t)$ and $y_{s k}(t)$ obtained by the Frog method.

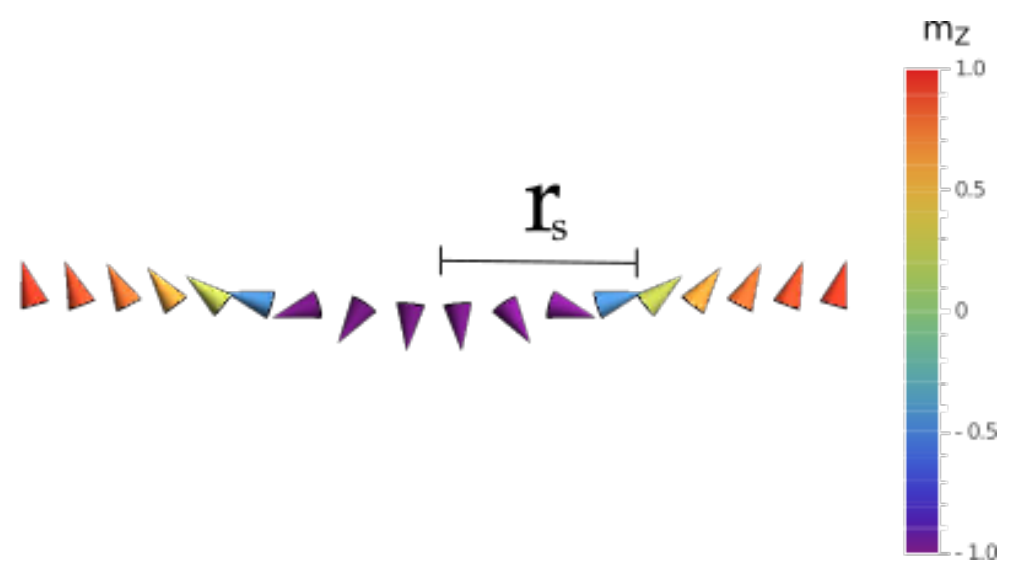

Figure 8- Schematic representation of the skyrmion radius $r_{s}$ definition. The radius $r_{s}$ is defined as the distance between the magnetic moment of the center of the skyrmion ( $-\boldsymbol{Z}$ direction) and the first magnetic moment with an angle of $90^{\circ}$ with respect to it. 
Figure 9 shows the behavior of the skyrmion radius as a function of its position $y_{s k}$, during the dynamics. We can observe that $r_{s}$ decreases as the skyrmion approaches the side edge, as can also be seen in Figure 4 and Figure 6.

By the continuous theoretical model [16], skyrmions have a topological charge of module $S=$ 1. However, as we do a micromagnetic study, that is, we treat our system as a discrete model, we obtain $S<1$ values for the skyrmion topological charge module. In figure 10 we show the behavior of the topological charge $S$ during the dynamics. Similar to the observed with the skyrmion radius, the topological charge decreases when the skyrmion approaches the side edge of the nanotrack. This behavior is a consequence of the deformation of the skyrmion when approaching the edge, which reduces its area.

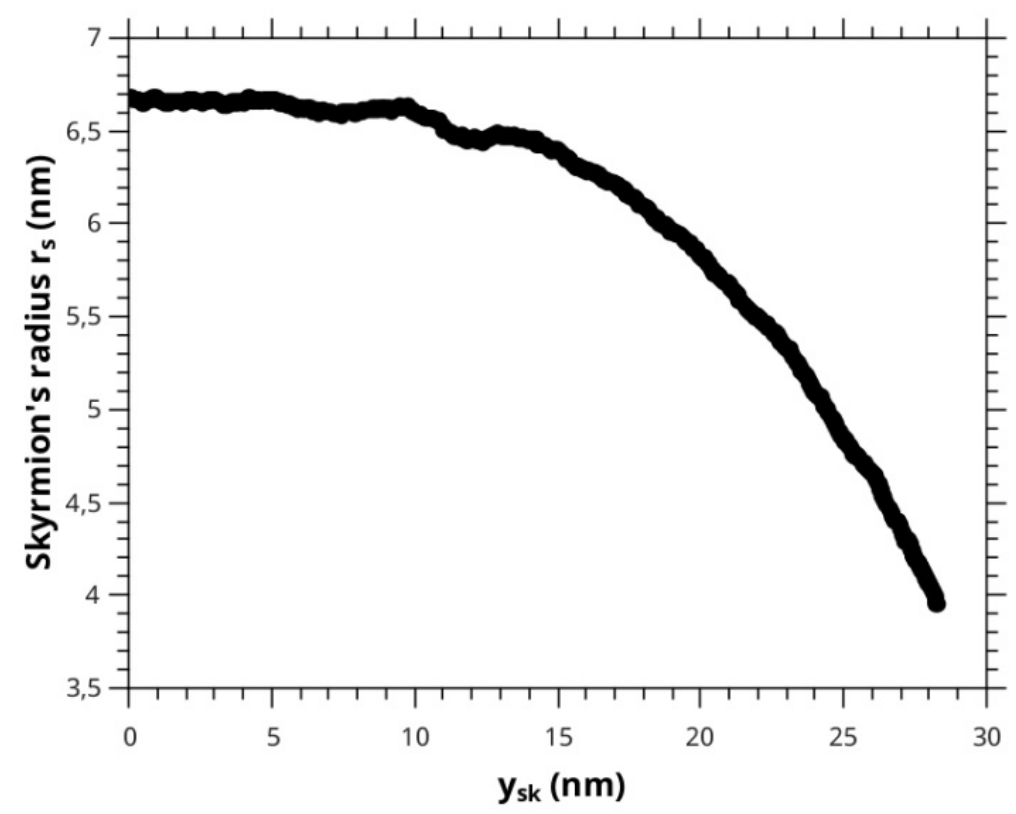

Figure 9- Skyrmion radius $\left(\boldsymbol{r}_{s}\right)$ behavior as a function of $\boldsymbol{y}_{\boldsymbol{s}}$ during the displacement. The $\boldsymbol{y}_{\boldsymbol{s}}$ is the $\boldsymbol{y}$ coordinate of the center of the skyrmion.

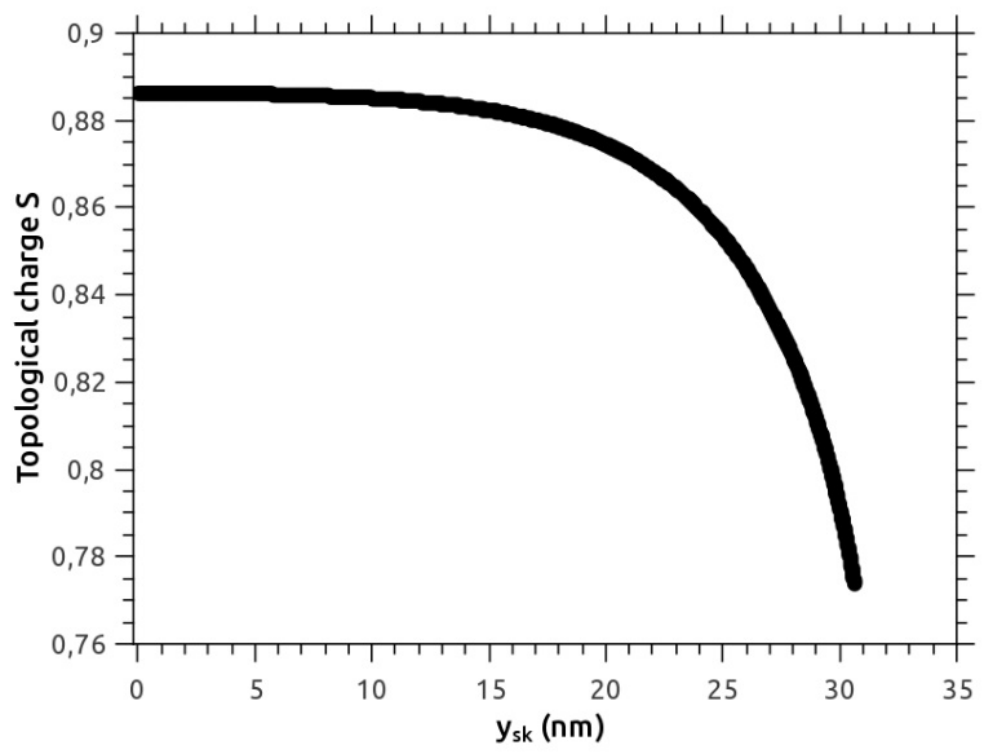

Figure 10- Topological charge $(\boldsymbol{S})$ behavior as a function of $\boldsymbol{y}_{\boldsymbol{s} \boldsymbol{k}}$ during the displacement. The $\boldsymbol{y}_{\boldsymbol{s} \boldsymbol{k}}$ is the $\boldsymbol{y}$ coordinate of the center of the skyrmion. 
Finally, we performed a study of the interaction energy $U\left(\boldsymbol{y}_{\text {sk }}\right)$ between the skyrmion and the side edge of the nanotrack. The interaction energy is a function of the $\boldsymbol{y}_{\boldsymbol{s} \boldsymbol{k}}$ coordinate of the center of the skyrmion. We consider the energy of the equilibrium configuration with the skyrmion located in the center of the nanotrack, as the reference energy $E_{0} . E_{0}$ is the energy of the configuration where $\boldsymbol{y}_{s k}=0$. We define $E\left(\boldsymbol{y}_{s k}\right)$ as the energy of the configuration where the center of the skyrmion has coordinate $\boldsymbol{y}_{\boldsymbol{s} \boldsymbol{k}} . E_{0}$ and $E\left(\boldsymbol{y}_{\boldsymbol{s} \boldsymbol{k}}\right)$ are calculated using the expression (1). The interaction energy has been estimated using the following expression:

$$
U\left(\boldsymbol{y}_{\boldsymbol{s}}\right)=E\left(\boldsymbol{y}_{\boldsymbol{s}}\right)-E_{0}
$$

Figure 11 shows the behavior of the interaction energy $U\left(\boldsymbol{y}_{s k}\right)$ between the skyrmion and the side edge, as a function of the $\boldsymbol{y}_{\boldsymbol{s}}$ coordinate of the center of the skyrmion. As the skyrmions approaches the edge, we can see that the interaction energy remains positive and increases until reaching a critical value at a position $y_{c} \approx 28.5 \mathrm{~nm}$. We can conclude that the skyrmion- edge interaction, up to that critical point, is repulsive. If the skyrmion exceeds this critical position $y_{c}$, it will be attracted and annihilated at the edge. This is probably due to the fact that the skyrmion for $\boldsymbol{y}_{\boldsymbol{s}}>y_{c}$, reaches a critical size that makes it unstable. This information is of crucial importance for the transport of skyrmions in nanotracks and their consequent technological application in spintronics. For example, this repulsion allows the skyrmion to travel through the nanotrack without escaping from the side edge, as long as the applied current is not sufficient for it to reach the $\boldsymbol{y}_{\boldsymbol{s} \boldsymbol{k}}>y_{c}$ region, where it will be annihilated.

To study the influence of the interaction energy on the skyrmion dynamics, we performed simulations to obtain the limit value $j_{c}$ of the applied current density that keeps the skyrmion in the $y_{s k}<$ $y_{c}$ region. We kept the width $W=80 \mathrm{~nm}$ and the thickness $T=2 \mathrm{~nm}$ but we considered the nanotrack with length $L=1.5 \mu \mathrm{m}$. Here we consider the origin of the coordinate system at the right edge of the nanotrack and the initial position of the skyrmion at this point $(x=0, y=0)$.

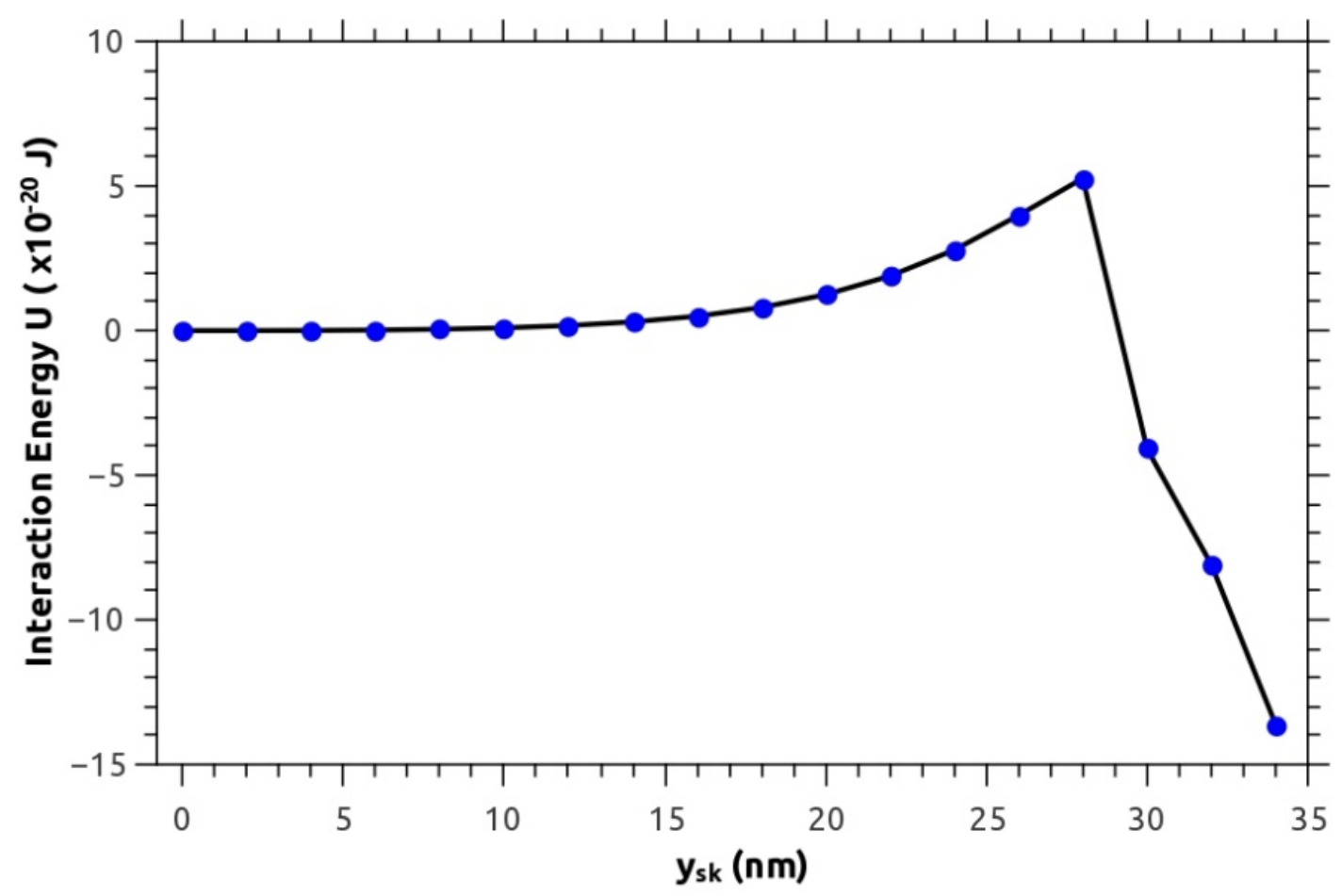

Figure 11- Interaction energy $\boldsymbol{U}\left(\boldsymbol{y}_{\boldsymbol{s}}\right)$ between the skyrmion and the side edge, as a function of the $\boldsymbol{y}_{\boldsymbol{s} \boldsymbol{k}}$ coordinate of the center of the skyrmion. 
Figure 12 shows the trajectories of the skyrmion during its displacement to 7 different values for the applied current density $j_{e}$. We can see that to keep the skyrmion on the nanotrack the limit value of the applied current density is $j_{c}=0.42 \times 10^{12} \mathrm{~A} / \mathrm{m}^{2}$. For $j_{e}<j_{c}$ the skyrmion reaches a stable position $y_{s k}<y_{c}$ and for $j_{e}>j_{c}$, the skyrmion reaches $y_{s k}>y_{c}$ region and is annihilated near the edge.

Our results for interaction energy qualitatively agree with the theoretical results of the reference [58] and this behavior for the skyrmion/edge interaction was also theoretically predicted for chiral magnets; see reference [59] .

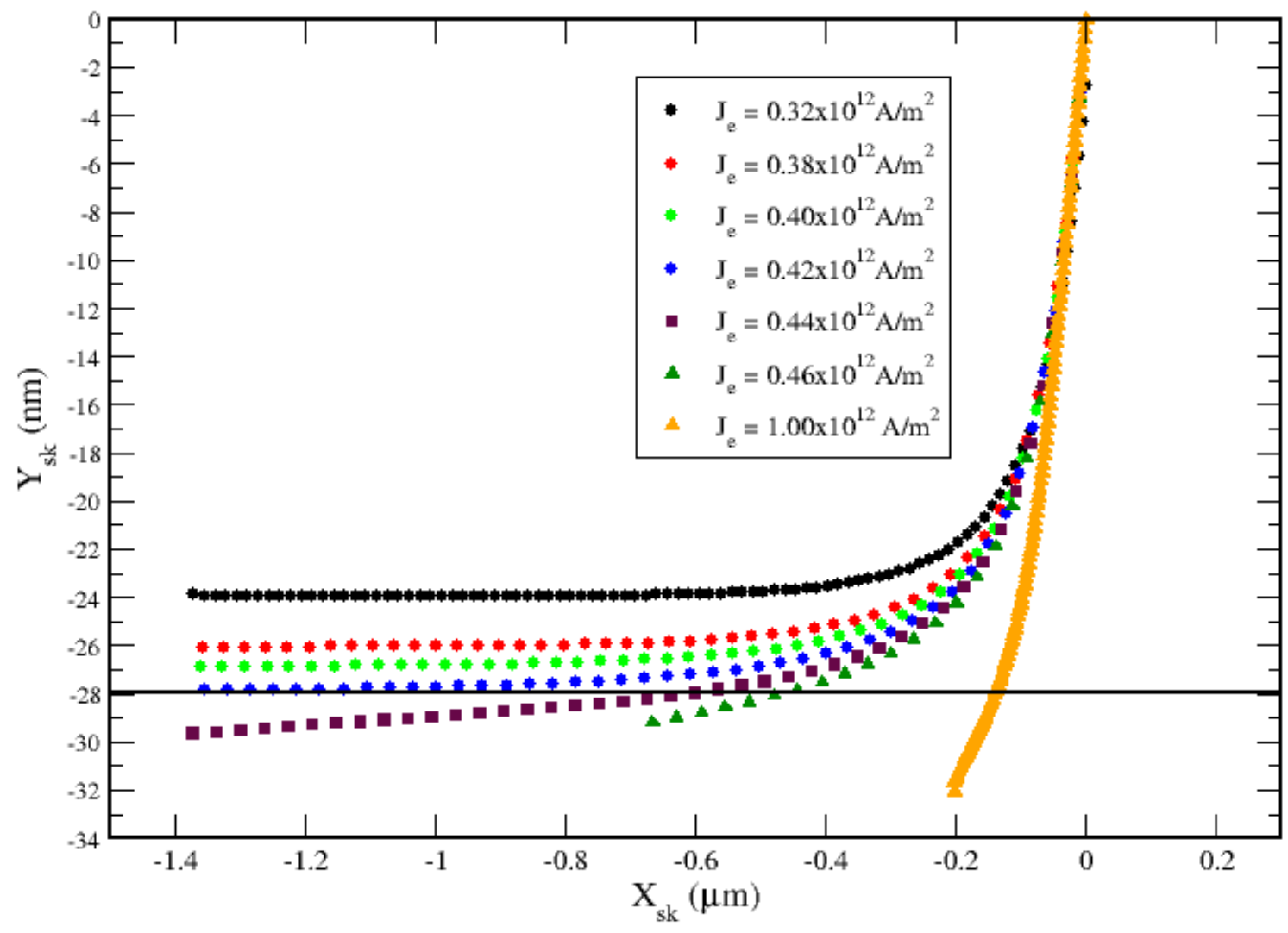

Figure 12 - Skyrmion trajectories in a nanotrack with width $W=80 \mathrm{~nm}$, thickness $T=2 \mathrm{~nm}$ and length $L=$ $1.5 \mu \mathrm{m}$. We consider 7 values for the applied current density $j_{e}$. We highlight the critical position $\quad y_{c} \approx$ $-28.0 \mathrm{~nm}$. The origin of the coordinate system is considered at the right edge of the nanotrack. 


\section{Conclusions}

In summary, the skyrmions will probably be the information carriers of the next generation of the data storage and logic devices. Because of this, it is important to systematically study the characteristics and behavior of structural properties of skyrmions during their dynamics, for future applications in spintronic devices.

In this work we performed micromagnetic simulations to study the skyrmion dynamics in nanotracks made of $\mathrm{Co} / \mathrm{Pt}$, submitted to the application of spin polarized current. We observed that the skyrmions suffer transverse deflection in their movements, as predicted in the literature. We basically use three methods to determine the position of the skyrmion during its dynamics: the Average Magnetization Method (Av), the Charge Method (s) and the Frog Method (Frog). We observed that the trajectory generated by the Frog Method is the closest to the displacement of the center of the skyrmion. We conclude that the Frog Method is the most accurate to track the skyrmion during dynamics providing in addition to position, data such as radius and topological charge. We analyzed the behavior of the $v_{x}$ and $v_{y}$ components of the skyrmion velocity during the movement and observed that as it approaches the edge, $\boldsymbol{v}_{\boldsymbol{x}}$ increases and $v_{y}$ decreases. We also observed changes in the structure of the skyrmion when it approaches the side edge, such as in the radius size and in the topological charge. We observed that both the radius and the topological charge decrease.

Completing our work, we performed a study of the interaction energy between the skyrmion and the side edge of the nanotrack. We observed that the skyrmion-edge interaction is repulsive to $y_{s k}<$ $y_{c}$. If the skyrmion exceeds this critical position, it will be attracted and annihilated at the edge. For the nanotrack considered in our study, we performed simulations to obtain the limit value $j_{c}$ of the applied current density that keeps the skyrmion in the $y_{s k}<y_{c}$ region.

From a technological point of view for possible applications in spintronic devices that use the transport of skyrmions on long nanotracks, the estimate of $j_{c}$ is of crucial importance. Using an applied current density $j_{e}<j_{c}$, the skyrmion can be transported on the nanotrack without escaping from the side edge.

\section{Acknowledgments}

This work was partially supported by CAPES, CNPq, FAPEMIG and FINEP (Brazilian Agencies). Numerical works were done at the Laboratório de Simulação Computacional do Departamento de Física da UFJF.

\section{References and Notes}

[1] Tehrani, S.; Slaughter, J. M.; Chen, E.; Durlam, M.; Shi, J.; and DeHerrera, M.; IEEE Trans. Magn, 1999, 35, 2814. https://doi.org/10.1109/20.800991

[2] Parkin, S. S. P.; Roche, K. P.; Samant, M. G.; Rice, P. M.; and Beyers, R. B.; J. Appl. Phys, 1999, 85, 5828. https://doi.org/10.1063/1.369932

[3] Gallagher, W. J.; and Parkin, S. S. P.; IBM J. Res. \& Dev, 2006, 50, 5. https://doi.org/10.1147/rd.501.0005

[4] Chappert, C.; Fert, A.; Van Dau, F.N.; Nat. Mate, 2007, 6 , 813. https://doi.org/10.1142/9789814287005 0015

[5] Fert, A.; Reyren, N.; Cros, V.; Nat. Rev. Mater, 2017, 2 , 17031.

https://doi.org/10.1038/natrevmats.2017.31

[6] Gomes, J. C. S.; Toscano, D.; Paixão, E. L. M.; Araujo, C. I. L. de; Sato, F.; Dias, R. A.; Coura, P. Z.; and Leonel, P. Z. AIP Advances, 2018, 8, 095017. https://doi.org/10.1063/1.5035258

[7] Araujo, C. I. L. de; Gomes, J. C. S.; Toscano, D.; Paixão, E. L. M.; Coura, P. Z.; Sato, F.; Massote, D. V. P.; and Leonel, S. A.; Appl. Phys. Lett, 2019, 114, 212403. https://doi.org/10.1063/1.5089949

[8] Kiselev, N.S.; Bogdanov, A.N.; Schäfer, R.; Rößler, U.K.; J. Phys. D: Appl. Phys, 2011, 44, $392001 . \quad$ https://doi.org/10.1088/0022$3727 / 44 / 39 / 392001$

[9] Skyrme, T. H. R.; Proc. R. Soc. London Ser. 1961, A $260, \quad 127$. https://doi.org/10.1142/97898127959220013

[10] Skyrme, T. H. R.; Nucl. Phys, 1962, 31, 556. https://doi.org/10.1016/0029-5582(62)90775-7

[11] Belavin, A.A.; Polyakov, A.M.; JETP Lett, 1975, 22, 245.

[12] Bogdanov, A.; Hubert, A.; J. Magn. Magn. Mater, $1994, \quad 138, \quad 255$. 
https://doi.org/10.1016/0304-8853(94)90046-9

[13] Kirakosyan, A.S.; Pokrovsky, V.L.; J. Magn. Magn. Mater, 2006, 305, 413. https://doi.org/10.1016/j.jmmm.2006.01.113

[14] Rößler, U.K.; Bogdanov, A.N.; Pfleiderer, C.; Nature, 2006, 442, 797. https://doi.org/10.1038/nature05056

[15] Sampaio, J.; Cros, V.; Rohart, S.; Thiaville, A.; Fert, A.; Nat. Nanotechnol, 2013, 8, 839. https://doi.org/10.1038/nnano.2013.210

[16] Nagaosa, N.; Tokura, Y.; Nat. Nanotechnol, 2013, 8 , 899.

https://doi.org/10.1038/nnano.2013.243

[17] Fert, A.; Cros V.; and Sampaio, J.; Nat. Nanotechnol, 2013, 8, 152. https://doi.org/10.1038/nnano.2013.29

[18] Mühlbauer, S.; Binz, B.; Jonietz, F.; Pfleiderer, C.; Rosch, A.; Neubauer, A.; Georgii, R. Böni, P.; Science, 2009, 323, 915. https://doi.org/10.1126/science.1166767

[19] Yu, X.Z.; Onose, Y.; Kanazawa, N.; Park, J.H.; Han, J.H.; Matsui, Y.; Nagaosa, N.; Tokura, Y.; Nature, 2010, 465, 901. https://doi.org/10.1038/nature09124

[20] Romming, N.; Hanneken, C.; Menzel, M.; Bickel, J.E.; Wolter, B.; Bergmann, K. von; Kubetzka, A.; Wiesendanger, R.; Science, 2013, 341, 636. https://doi.org/10.1126/science.1240573

[21] Zhang, S; Zhang, J; Zhang, Q; Barton, C; Neu, V; Zhao, Y; $\underline{\text { Hou }}, \quad Z ; \underline{\text { Wen, }}$ Y; Gong, C; Kazakova, O; Wang, W; Peng, Y; Garanin, D. A; Chudnovsky, E, M; and Zhang, X; Appl. Phys. Lett. 2018, 112, 132405. https://doi.org/10.1063/1.5021172

[22] Chen, G.; Mascaraque, A.; N'Diaye, A.T.; Schmid, A.K.; Appl. Phys. Lett. 2015, 106, 242404. https://doi.org/10.1063/1.4922726

[23] Woo, S. ; Litzius, K.; Krüger, B.; Im, M.-Y.; Caretta, L.; Richter, K.; Mann, M.; Krone, A.; Reeve, R.M.; Weigand, M.; Agrawal, P.; Lemesh, I.; Mawass, M.-A.; Fischer, P.; Kläui, M.; G.S.D. Beach, Nat. Mater. 2016, 15, 501. https://doi.org/10.1038/nmat4593

[24] Boulle, O.; Vogel, J.; Yang, H.; Pizzini, S.; Chaves, D.S.; Locatelli, A.; Mentes, T.O.; Sala, A.; Buda-Prejbeanu, L.D.; Klein, O.; Belmeguenai, M.;
Roussigné, Y.; Stashkevich, A.; Chérif, S.M.; Aballe, L.; Foerster, M.; Chshiev, M.; Auffret, S.; Miron, I.M.; Gaudin, G.; Nat. Nanotechnol. 2016, 11 ,

https://scholarbank.nus.edu.sg/handle/10635/1428 $\underline{23}$

[25] He, M.; Peng, L.; Zhu, Z.; Li, G.; Cai, J.; Li, J.; Wei, H.; Gu, L.; Wang, S.; Zhao, T.; Shen, B.; Zhang, Y.; Appl. Phys. Lett. 2017, 111, 202403. https://doi.org/10.1063/1.5001322

[26] Dzyaloshinsky, I.; J. Phys. Chem. Solids, 1958, 4 , $241 . \quad$ https://doi.org/10.1016/0022$\underline{3697(58) 90076-3}$

[27] Moriya, T.; Phys.Rev. 1960,120, 91. https://doi.org/10.1103/PhysRev.120.91

[28] Crépieux, A.; Lacroix, C.; J. Magn. Magn. Mater. 1998, 182, 341. https://doi.org/10.1016/S0304-8853(97)01044-5

[29] Hu, C.D.; J. Phys.: Condens.Matter. 2012, 24, $086001 . \quad$ https://doi.org/ 10.1088/09538984/24/8/086001

[30] Purnama, I.; Gan, W. L.; Wong, D. W.; Lew, W. S.; Sci. Rep. 2015, 5, 10620. https://doi.org/10.1038/srep10620

[31] Jiang, W.; Zhang, X.; Yu, G.; Zhang, W.; Wang, X.; Jungfleisch, M. B.; Pearson, J. E.; Cheng, X.; Heinonen, O.; Wang, K. L.; Zhou, Y.; Hoffmann, A.; Velthuis, S. G. E. te; Nature Phys. 2017, 13, 162. https://doi.org/10.1038/nphys3883

[32] Zhang, X.; Zhou, Y.; Ezawa, M.; Nat. Commun. 2016, 7 , 10293.

https://doi.org/10.1038/ncomms10293

[33] Toscano, D.; Leonel, S.A.; Coura, P.Z.; Sato, F.; Dias, R.A.; Costa, B.V.; Appl. Phys. Lett. 2012, 101, 252402. https://doi.org/10.1063/1.4772071

[34] Toscano, D.; Leonel, S.A.; Coura, P.Z.; Sato, F.; Costa, B.V.; Vázquez, M.; J. Magn. Magn. Mater. 2016, 419, 37. https://doi.org/10.1016/j.jmmm.2016.05.107

[35] Burn, D.M.; Atkinson, D.; J. Appl. Phys. 2014, 116, 163901. https://doi.org/10.1063/1.4900437

[36] Sapozhnikov, M.V.; Vdovichev, S.N.; Ermolaeva, O.L.; Gusev, N.S.; Fraerman, A.A.; Gusev, S.A.; Petrov, Yu.V.; Appl. Phys. Lett. 2016, 109, 042406. https://doi.org/10.1063/1.4958300.

[37] Ozkaya L, D.; Langford, R. M.; Chan, W. L.; Petford-Long, A. K.; J. Appl. Phys. 2002, 91, 9937. 
https://doi.org/10.1063/1.1477265

[38] Moreau-Luchaire, C.; Moutafis, C.; Reyren, N.; Sampaio, J.; Vaz, C. A. F.; Horne, N. Van; Bouzehouane, K.; Garcia, K.; Deranlot, C.; Warnicke, P.; Wohlhüter, P.; George, J.-M.; Weigand, M.; Raabe, J.; Cros, V.; Fert, A.; Nat. Nanotechnol. 2016, 11, 444. https://doi.org/10.1038/nnano.2015.313

[39] Yang, H.; Thiaville, A.; Rohart, S.; Fert, A.; Chshiev, M.; Phys. Rev. Lett., 2015, 115267210.

[40] Legrand, W.; Maccariello, D.; Reyren, N.; Garcia, K.; Moutafis, C.; Moreau-Luchaire, C.;

Collin, S.; Bouzehouane, K.; Cros, V.; Fert, A.; Nano Lett., 2017, 17, 2703-2712.

[41] Paixão, E. L. M.; Toscano, D.; Gomes, J. C. S.; Monteiro Jr., M. G.; Sato, F.; Leonel, S. A.; Coura, P. Z.; J. Magn. Magn. Mater. 2018, 451, 639. https://doi.org/10.1016/j.jmmm.2017.11.117

[42] Silva, J. H.; Toscano, D.; Sato, F.; Coura, P. Z.; Costa, B. V.; Leonel, S. A.; J. Magn. Magn. Mater. 2012, 324 , 3083.

https://doi.org/10.1016/j.jmmm.2012.05.006

[43] Toscano, D.; Leonel, S. A.; Coura, P. Z.; Sato, F.; J. Magn. Magn. Mater. 2019, 480, 171. https://doi.org/10.1016/j.jmmm.2019.02.075

[44] Toscano, D.; Mendonça, J.P.A.; Miranda, A.L.S.; Araujo, C.I.L. de; Sato, F.; Coura, P. Z.; Leonel, S. A.; J. Magn. Magn. Mater. 2020, 504, 166655.

https://doi.org/10.1016/j.jmmm.2020.166655

[45] Shen, L.; Li, X.; Zhao, Y.; Xia, J.; Zhao, G.; Zhou, Y.; Phys. Rev. Applied 2019, 12, 064033. https://doi.org/10.1103/PhysRevApplied.12.064033

[46] Zhang, X.; Zhou, Y.; Ezawa, M.; Sci. Rep. 2016, 6, 24795. https://doi.org/10.1038/srep24795

[47] Barker, J.; Tretiakov, O. A.; Phys. Rev. Lett. 2016, 116, 147203. https://doi.org/10.1103/PhysRevLett.116.147203
[48] Toscano, D. ; Santece, I. A.; Guedes, R. C. O.; Assis, H. S.; Miranda, A. L. S.; Araujo, C. I. L. de; Sato, F.; Coura, P. Z.; Leonel, S. A.; Journal of Applied Physics, 2020, 127, 193902. https://doi.org/10.1063/5.0006219

[49] Mendonça, J.P.A.; Santece, I.A.; Monteiro Jr., M.G.; Leonel, S.A.; Coura, P.Z.; Sato, F.; arXiv: 1906.11375,

2019.

https://arxiv.org/abs/1906.11375

[50] Suessa, D.; Fidlera, J.; Schrefl, T.; Handbook of Magnetic Materials, 2006, 16, 41.

[51] Zhang, S. ; ; Li, Z.; Phys. Rev. Lett. 2004, 93, 127204.

https://doi.org/10.1103/PhysRevLett.93.127204

[52] Fook, H. T.; Gan, W. L.; Lew, W. S.; Sci. Rep. 2016, 6, 21099. https://doi.org/10.1038/srep21099

[53] Thiaville, A.; Nakatani, Y.; Miltat, J.; Suzuki, Y.; Europhys. Lett. 2005, 69, 990. https://doi.org/10.1209/epl/i2004-10452-6

[54] Yuan, H. Y.; Wang, X. R.; Phys. Rev. 2015, B 92,

054419.

https://doi.org/10.1103/PhysRevB.92.054419

[55] Zhang, X.; Xia, J.; Zhao, G. P.; Liu, X.; Zhou, Y.; IEEE Trans. Magn. 2016, 53, 1500206. https://doi.org/10.1109/TMAG.2016.2641384

[56] Papanicolaou; N.; Tomaras, T.; Nuclear Physics B, 1991, 360. 2-3, 425. https://doi.org/10.1016/0550-3213(91)90410-Y

[57] Moutafis, C.; Komineas, S.; Bland, J. A. C.; Phys. Rev. B, 2009, 79, 224429. https://doi.org/10.1103/PhysRevB.79.224429

[58] Martineza, J.C; Lewb, W.S.; Ganb, W.L.; Jalil, M.B.A.; J. Magn. Magn. Mater., 2018, 465, 685. https://doi.org/10.1016/j.jmmm.2018.06.031

[59] Iwasaki, J.; Mochizuki, M.; and Nagaosa, N.; Nat. Nanotechnol. 2013, 8, 742. https://doi.org/10.1038/NNANO.2013.176 